CASE REPORT

\title{
Accurate diagnosis of chronic low back pain in a high-level college athlete : a case report
}

\author{
Kenji Yokoyama, Kazuta Yamashita, Masatoshi Morimoto, Fumitake Tezuka, Fumio Hayashi, Yoichiro Takata, \\ Toshinori Sakai, Kosaku Higashino, Takashi Chikawa, Hiroshi Yonezu, Akihiro Nagamachi, and Koichi Sairyo \\ Department of Orthopedics, Tokushima University Hospital, Tokushima, Japan
}

\begin{abstract}
A 21 -year-old woman who was high-level college softball player presented with a 6 -month history of low back pain that had been treated unsuccessfully by medication at local clinics. There was tenderness in the left paravertebral muscle at the lower lumbar level. X-ray and computed tomography revealed congenital scoliosis and an L6 hemivertebra. Short tau inversion recovery magnetic resonance imaging showed a fluid collection at the left L6-S1 facet joint. We performed a diagnostic facet injection, consisting of $1 \%$ lidocaine and steroids, that was infiltrated into the left facet joint at L6-S1. Her persistent low back pain disappeared immediately after the facet block. We diagnosed left-sided facet arthritis at L6-S1. Due to her multiple congenital anomalies, excessive loading occurred at the facet joint. Therefore, we opted for conservative management, including mobilization of the thoracic spine and stretching of hamstrings and quadriceps. This case report underscores the importance of accurate diagnosis of low back pain and of the diagnostic utility of short tau inversion recovery magnetic resonance imaging and lumbar facet block in young athletes with chronic spinal pain. J. Med. Invest. 64 : 313-316, August, 2017
\end{abstract}

Keywords : low back pain, diagnosis, facet joint, athlete, MRI

\section{INTRODUCTION}

Low back pain is a serious problem for adolescent athletes. The incidence of low back pain among athletes in this age group has been reported to be in the range of $5 \%-66 \%(1-5)$. The main cause of specific low back pain is spondylolysis/spondylolisthesis. Disk-related problems are relatively uncommon in children (1). Infection, inflammation, and tumor are less common causes (6). On the other hand, the most common causes of nonspecific low back pain are musculoskeletal conditions, such as hyperlordosis, sprains, and strains $(6,7)$. Musculoskeletal problems account for at least $50 \%$ of cases of nonspecific low back pain (7-10).

Diagnostic modalities available for evaluation of back pain in children include plain radiograph, computed tomography (CT), magnetic resonance imaging (MRI), single-photon emission CT, and laboratory investigations (10). However, a specific generator of low back pain is not always found, which makes diagnosis and treatment more difficult (11). One study concluded that exhaustive diagnostic protocols may not be necessary in pediatric patients with low back pain because their symptoms do not permit a definitive diagnosis (10). However, although most cases are self-limiting, some young athletes have persistent symptoms (6). Moreover, low back pain might reduce their athletic performance. Accurate diagnosis and adequate treatment of low back pain is very important in adolescent athletes.

This case report describes the presentation of a young female athlete with low back pain, highlights the importance of accurate diagnosis, and demonstrates the diagnostic utility of facet joint injections in young patients with chronic spinal pain.

Received for publication April 24, 2017 ; accepted July 12, 2017.

Address correspondence and reprint requests to Kazuta Yamashita, MD, Tokushima University Graduate School of Medicine, 3-18-15 Kuramoto, Tokushima, Tokushima 770-8503, Japan and Fax : +81-88633-0178.

\section{CASE REPORT}

The patient was a 21-year-old college student and a high-level right-handed softball pitcher. She had a 6 -month history of low back pain, which was present during sports activity. The pain had gradually increased to the point where she had been unable to participate in sporting activity for 4 months. She was treated unsuccessfully by medication (nonsteroidal anti-inflammatory drugs, serotonin-norepinephrine reuptake inhibitors, tramadol) at local clinics. The patient's medical history included congenital scoliosis but there was no family history of scoliosis.

Examination revealed tenderness of the left lower lumbar paravertebral muscles. Her pain increased in extension but not in flexion. Her neurologic examinations, including manual muscle, sensory, and deep tendon reflex tests, were all normal.

Plain radiographs revealed congenital scoliosis with left-sided hemivertebra at L6 (Figure 1). The L3 and L4 vertebrae showed congenital fusion. Dynamic radiographs indicated hypermobility at the lumbosacral joint (Figure 2). Three-dimensional CT scans revealed congenital union of hemivertebrae at L5 and L6 (Figure 3). A posterior view on three-dimensional CT images clearly indicated multilevel dysplasia of the facet joints and spina bifida at L4. T2-weighted MRI revealed low-grade disc degeneration at the lumbosacral junction (Figure 4). Coronal short tau inversion recovery (STIR) MRI showed afluid collection at the L6-S1 facet joint on the left side. Axial STIR MRI views also indicated joint fluid at the same level (Figure 5). A diagnosis of left-sided facet arthritis at L6-S1 was made.

We then proceeded to diagnostic facet injection. Xylocaine $1 \%$ and a steroid were infiltrated into the left facet joint at L6-S1. Her low back pain, which had persisted for more than 6 months, disappeared immediately but returned 2 days after the block.

A conservative management plan was started involving mobilization of the thoracic spine and hip joint, including stretching of the hamstrings and quadriceps, because the imbalance of the spine 


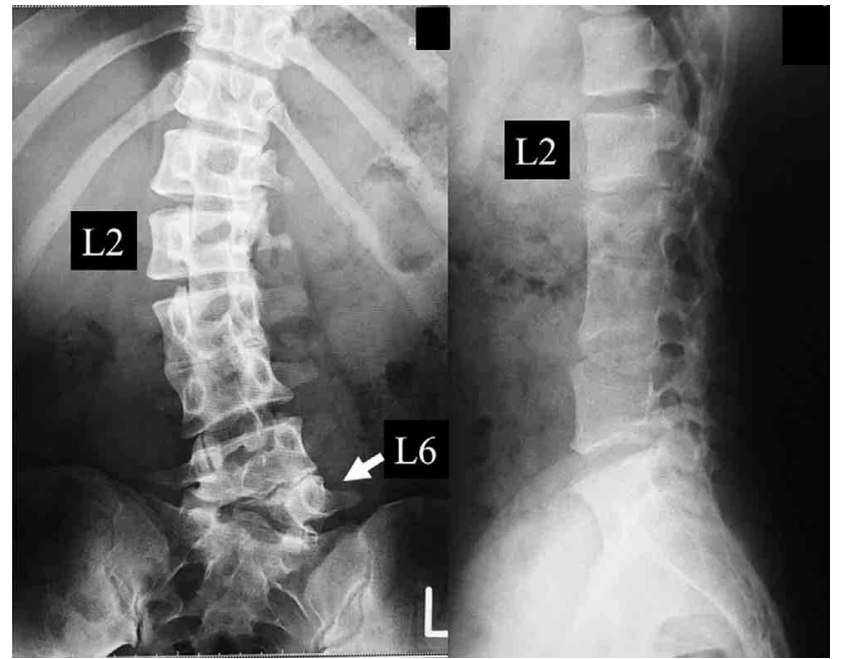

Figure 1. Plain radiographs of the lumbar spine. Anteroposterior (left panel) and lateral (right panel) views showing congenital scoliosis with left-sided hemivertebra at L6 and congenital fusion of the L3 and L4 vertebrae.

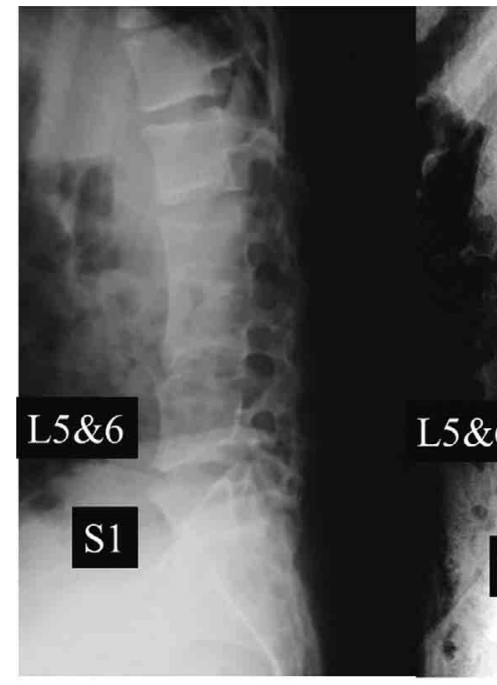

Flexion

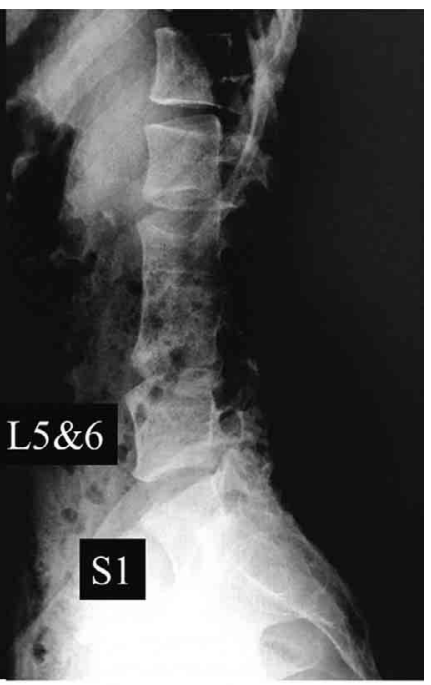

Extension
Figure 2. Dynamic radiographs of the lumbar spine.

The left panel shows a lateral view of lumbar flexion. The right panel shows a lateral view of lumbar extension. Hypermobility at the lumbosacral joint is shown.

due to multiple congenital anomalies, including a fused spine, caused excessive loading at the facet joints. This strategy was based on the joint-by-joint approach proposed by Cook (12). After the conservative therapy, this patient's low back pain gradually improved, and she has not required oral medicine at all. Finally, she had returned to play softball as her competitive level at 6 months after her consultation to our hospital.

\section{DISCUSSION}

Low back pain is a serious and common problem in athletes.

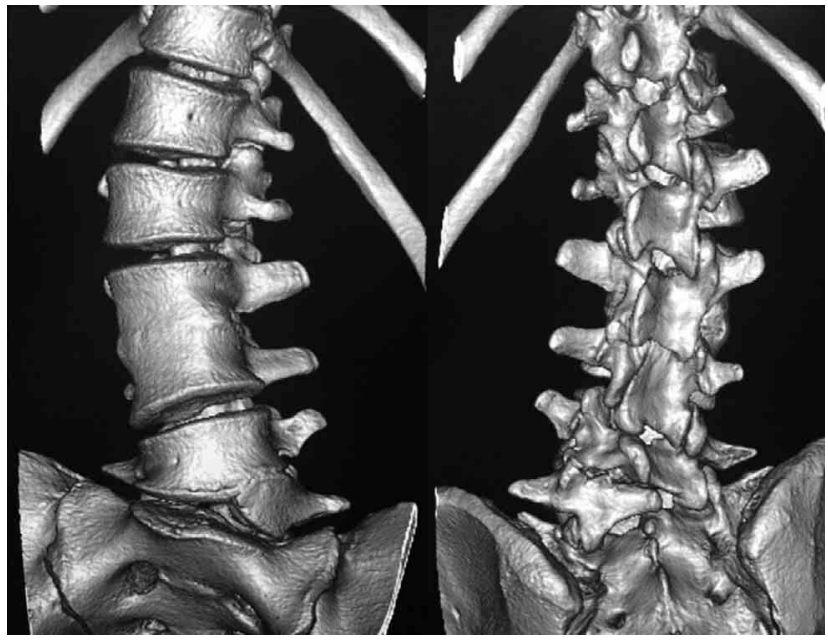

Figure 3. Three-dimensional computed tomography scans of the lumbar spine.

The left panel shows an anterior view and the right panel shows a posterior view. Congenital fusion of L5 and L6 hemivertebrae, multilevel dysplasia of the facet joints, and $\mathrm{L} 4$ spina bifida are shown.

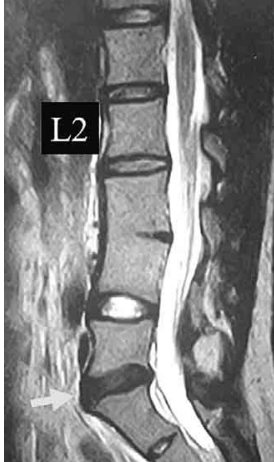

T2-WI mid-sagittal

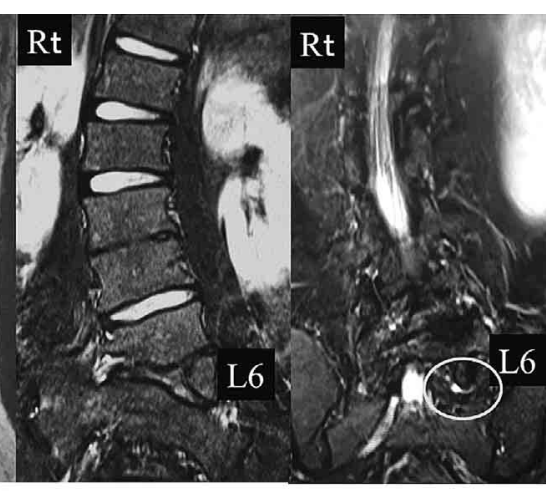

STIR coronal
Figure 4. Magnetic resonance images of the lumbar spine.

The left panel shows a mid-sagittal T2-weighted image. The middle and right panels show a coronal STIR image. The arrow indicates low-grade disc degeneration at the lumbosacral junction. The circle indicates a fluid collection at the left L6-S1 facet joint. Abbreviation : STIR, short tau inversion recovery

However, it was difficult to diagnose the exact cause of low back pain in this patient. This case highlights the importance of STIR MRI and diagnostic facet injection.

The diagnostic utility of STIR MRI is optimal for ankylosing spondylitis (13), pedicle fracture and degenerative facet joints (14), and spondylolysis $(15,16)$. Fluid collection, which is an indicator of inflammatory events, and bone marrow lesions, which could be micro bleeding in the bone marrow, can be evaluated on STIR MRI (17). In our case, STIR MRI showed a high-intensity change at the left L6-S1 facet joint. Therefore, we suspected that inflammation at this site was the cause of her symptoms and proceeded to a lumbar facet joint injection.

Some studies have suggested that facet joint injection is a reasonable intervention for chronic low back pain (18-20). There is level I 


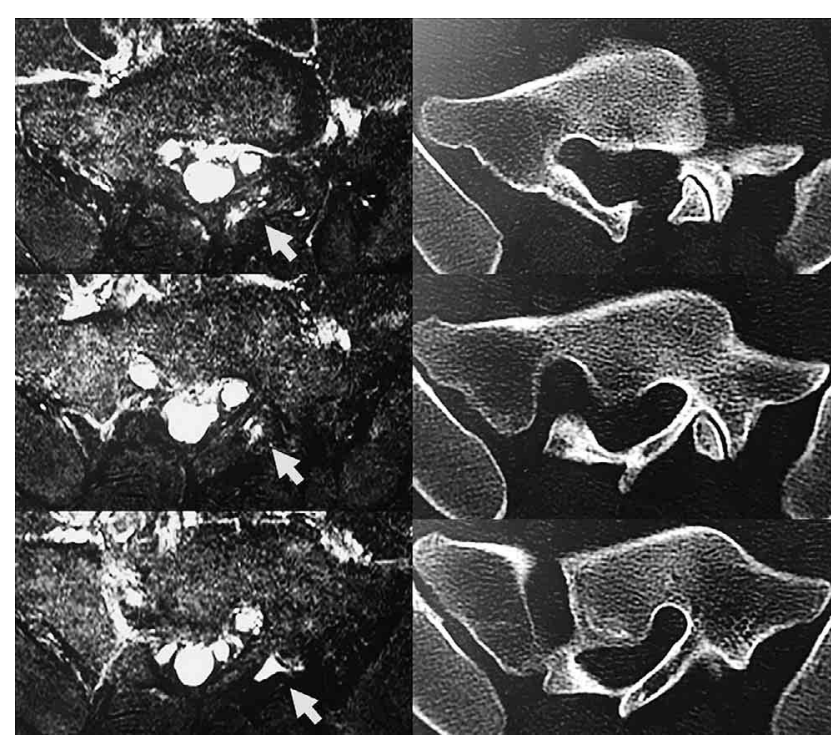

Figure 5. Axial images of L6-S1 on short tau inversion recovery magnetic resonance imaging (left panel) and computed tomography (right panel).

The arrows indicate a fluid collection in the joint on the left side.

evidence for the diagnostic validity of lumbar facet joint injection in that it decreases pain by $75 \%$ and allows patients to perform previously painful movements. For therapeutic interventions, the evidence varies from level II to level III (18). Temporary relief of low back pain was achieved by diagnostic and therapeutic facet joint injection in our patient. A multicenter randomized controlled trial in Japan shows the exercise performed trunk muscle strengthening and stretching was more effective than nonsteroidal antiinflammatory drugs for Japanese patients with chronic low back pain (21). Therefore, we were convinced that this was the source of the patient's low back pain and she was treated by physical therapy to mobilize the thoracic spine and hip joint.

One study concluded that facet joint injection containing lidocaine $0.125 \%-1.0 \%$ is toxic to the chondrocytes implicated in the development of osteoarthritis (22). However, our patient received only one facet joint injection because we considered that progression of her facet joint osteoarthritis was less likely than an adverse event associated with repeated facet joint injection.

\section{CONCLUSION}

An exact diagnosis is needed in young athletes suffering from low back pain. STIR MRI and lumbar facet injection are useful modalities for both clinicians and athletes.

\section{CONFLICT OF INTEREST}

The authors declare that they have no competing interests.

\section{REFERENCES}

1. Purcell L, Micheli L: Low back pain in young athletes. Sports Health $1: 212-222,2009$

2. Daniels JM, Pontius G, El-Amin S, Gabriel K : Evaluation of low back pain in athletes. Sports Health $3: 336-345,2011$
3. Mueller S, Mueller J, Stoll J, Prieske O, Cassel M, Mayer F : Incidence of back pain in adolescent athletes : a prospective study. BMC Sports Sci Med Rehabil 7 ; 8 :38. eCollection, 2016

4. Moradi V, Memari AH, ShayestehFar M, Kordi R: Low Back Pain in Athletes Is Associated with General and Sport Specific Risk Factors : A Comprehensive Review of Longitudinal Studies. Rehabil Res Pract : 850184, 2015

5. Müller J, Müller S, Stoll J, Fröhlich K, Otto C, Mayer F : Back pain prevalence in adolescent athletes. Scand J Med Sci Sports 27(4) : 448-454, 2017

6. Bono CM : Low-back pain in athletes. J Bone Joint Surg Am $86: 382-396,2004$

7. Kim HJ, Green DW : Adolescent back pain. Curr Opin Pediatr $20: 37-45,2008$

8. Combs JA, Caskey PM : Back pain in children and adolescents : a retrospective review of 648 patients. South Med J 90 : 789-792, 1997

9. Feldman DS, Hedden DM, Wright JG : The use of bone scan to investigate back pain in children and adolescents. J Pediatr Orthop $20: 790-795,2000$

10. Bhatia NN, Chow G, Timon SJ, Watts HG : Diagnostic modalities for the evaluation of pediatric back pain : a prospective study. J Pediatr Orthop 28 : 230-233, 2008

11. Nachemson AL : Newest knowledge of low back pain. A critical look. Clin Orthop Relat Res. 279: 8-20, 1992

12. Cook G, Burton L, Hoogenboom BJ, Voight M : Functional movement screening : the use of fundamental movements as an assessment of function - part 1. Int J Sports Phys Ther 9(3) : 396-409, 2014

13. Weber U, Hodler J, Kubik RA, Rufibach K, Lambert RG, Kissling RO, Pfirrmann CW, Maksymowych WP : Sensitivity and specificity of spinal inflammatory lesions assessed by whole-body magnetic resonance imaging in patients with ankylosing spondylitis or recent-onset inflammatory back pain. Arthritis Care Res 61: 900-908, 2009

14. Borg B, Modic MT, Obuchowski N, Cheah G : Pedicle marrow signal hyperintensity on short tau inversion recovery- and T2weighted images : Prevalence and relationship to clinical symptoms. Am J Neuroradiol 32 : 1624-1631, 2011

15. Sairyo K, Sakai T, Mase Y, Kon T, Shibuya I, Kanamori Y, Kosugi T, Dezaw A : Painful lumbar spondylolysis among pediatric sports players : a pilot MRI study. Archives of Orthopaedic and Trauma Surgery 131(11) : 1485-1489, 2011

16. Sairyo K, Katoh S, Takata Y, Terai T, Yasui N, Goel VK, Masuda A, Vadapalli S, Biyani A, Ebraheim N : MRI signal changes of the pedicle as an indicator for early diagnosis of spondylolysis in children and adolescents : a clinical and biomechanical study. Spine (Phila Pa 1976) 31(2) : 206-211, 2006

17. Sairyo K, Katoh S, Sasa T, Yasui N, Goel VK, Vadapalli S, Masuda A, Biyani A, Ebraheim N : Athletes with unilateral spondylolysis are at risk of stress fracture at the contralateral pedicle and pars interarticularis : a clinical and biomechanical study. Am J Sport Med 33 : 583-590, 2005

18. Manchikanti L, Hirsch JA, Falco FJE, Boswell MV : Management of lumbar zygapophysial (facet) joint pain. World J Orthop $7: 315-337,2016$

19. Falco FJ, Manchikanti L, Datta S, Sehgal N, Geffert S, Onyewu O, Zhu J, Coubarous S, Hameed M, Ward SP, Sharma M, Hameed H, Singh V, Boswell MV : An update of the effectiveness of therapeutic lumbar facet joint interventions. Pain Physician 15 : E909-953, 2012

20. Gorbach C, Schmid MR, Elfering A, Hodler J, Boos N : Therapeutic efficacy of facet joint blocks. Am J Roentgenol 186 : 1228-1233, 2006 
21. Shirado O, Doi T, Akai M, Hoshino Y, Fujino K, Hayashi K, Marui E, Iwaya T : Japan Low back-pain Exercise Therapy Study ; Investigators Japanese Orthopaedic Association ; Japanese Society for Musculoskeletal Rehabilitation ; Japanese Clinical Orthopaedic Association. Multicenter randomized controlled trial to evaluate the effect of home-based exercise on patients with chronic low back pain : the Japan low back pain exercise therapy study. Spine (Phila Pa 1976) 35(17) : E 811-819, 2010

22. Takeno K, Kobayashi S, Miyazaki T, Shimada S, Kubota M, Meir A, Urban J, Baba H : Lidocaine cytotoxicity to the zygapophysial joints in rabbits : changes in cell viability and proteoglycan metabolism in vitro. Spine (Phila Pa 1976) 34 : E 945-951, 2009 